\title{
Demenz und Ethik - Personen mit Demenz im Akutkrankenhaus
}

\author{
Der Mensch mit Demenz bleibt Person Soll der Umgang mit Menschen mit Demenz \\ menschenwürdig sein, müssen wir diese als Personen wahrnehmen und dürfen sie \\ nicht aus dem „Club der Personen“ ausschließen. Wir müssen uns über unsere eige- \\ nen Ängste und Formen der Abwehr hinausbewegen.
}

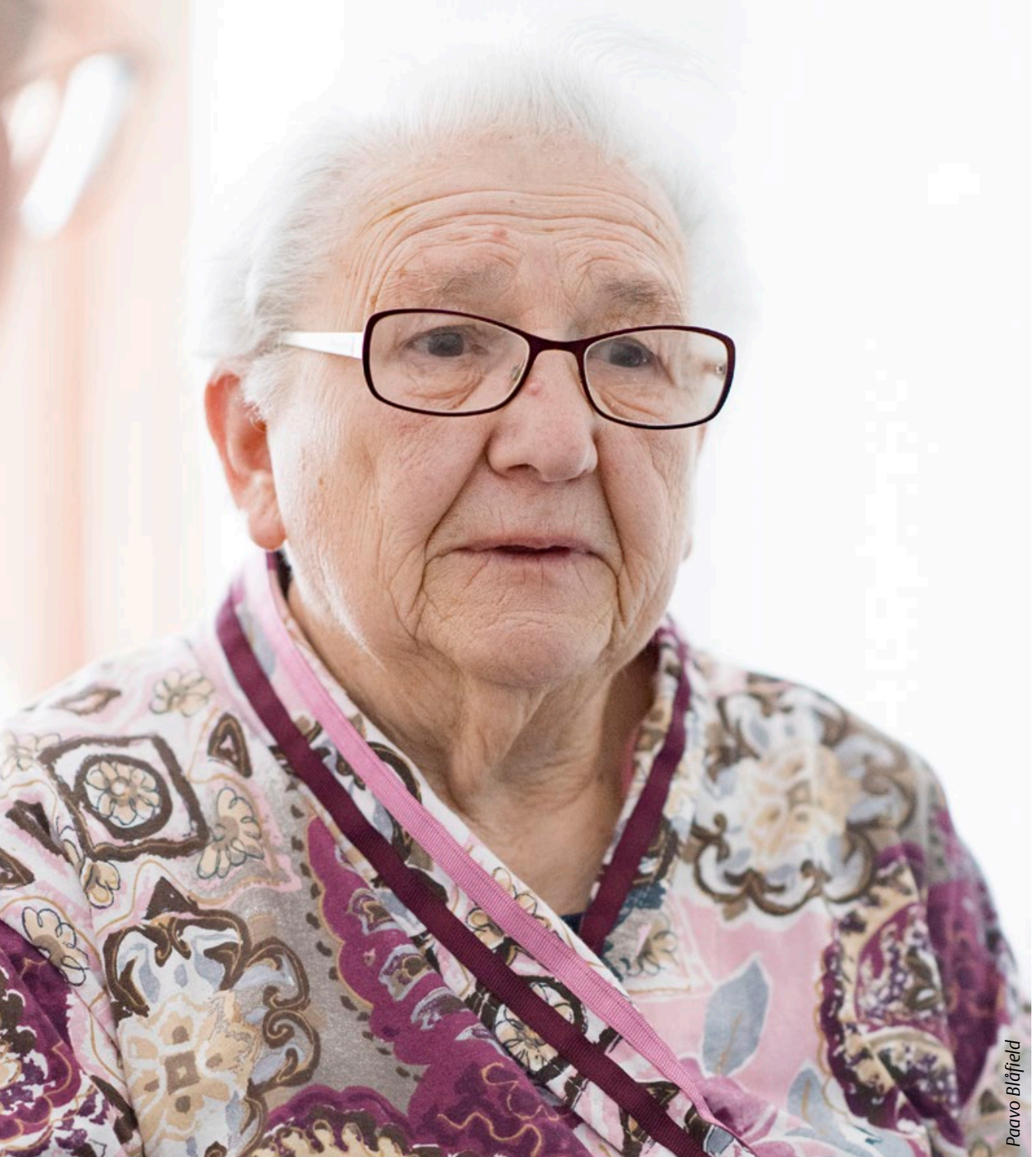

Ist sie aus dem „Club der Personen“ ausgeschlossen? (Symbolbild).
- Joachim Heil -

Die Ausgangslage

In Deutschland werden gegenwärtig jährlich mehr als 8 Millionen Patienten im Alter von über 65 Jahren beispielsweise wegen eines Knochenbruchs, eines Harnwegsinfekts oder einer Lungenentzündung stationär behandelt. Von diesen Patienten leiden $40 \%$ an kognitiven Beeinträchtigungen und $20 \%$ an einer Demenz, was bei der Aufnahme in den wenigsten Fällen bekannt ist. ${ }^{1}$

Im Verlauf eines stationären Aufenthalts kommt es häufig zu einem „weiteren Selbstständigkeitsverlust“, zur „Verschlechterung des kognitiven Status und zu einem vermehrten Auftreten problematischer Verhaltensweisen“ ${ }^{2}$ - insbesondere sog. „herausforderndem Verhalten“ (z.B. nächtliche Unruhe oder Verirren auf der Station sowie das Ablehnen von Nahrung) -, die oftmals eine wesentlich längere und mit hohen Kosten verbundene Aufenthaltsdauer zur Folge haben. ${ }^{3}$

Pflege zwischen Systemlogik und Lebenswelt

Die Ursachen einer Verschlechterung des kognitiven Status und eines vermehrten Auftretens von klinischen und verhaltensbedingten Komplikationen sind unter anderem das Fehlen vertrauter Bezugspersonen, eine veränderte und unübersichtliche Umgebung, starre ökonomische, vorwiegend an den Kranken- 
hausroutinen ausgerichtete Abläufe und nicht zuletzt das im „Spannungsfeld zwischen Systemlogik und Lebenswelt"4 gefangene ärztliche und pflegerische Personal.

\section{Die Fallgeschichte: 1. Szene (1. Teil) - Frau Mangold}

Krankenzimmer auf einer Station in einer Klinik. Frau Mangold, eine Patientin $(\mathrm{P})$ in reduziertem Allgemeinzustand, sitzt im Nachthemd auf der Bettkante. Sie hat einen Verband am rechten Unterarm. Schwester Maria (S), eine Krankenschwester, hilft ihr beim Anziehen der Hausschuhe.

P: „Ich weiß gar nicht, wie es weitergehen soll ...“

\section{S: „Nun mal ganz langsam!“ \\ P: „Keiner sagt mir etwas. Die Ärzte} ..."

S: (fällt ins Wort) „Sehen Sie, Frau Mangold, das kriegen wir schon hin.“

P: „Heute Nacht habe ich kaum geschlafen. Ich weiß gar nicht ..."

S: (unterbricht wieder; versucht, der Patientin den zweiten Schuh anzuziehen) „So, jetzt noch diesen Schuh.“

P: „Ich habe solche Angst. - Was wollen die denn mit mir machen?"

(Schwester Maria wendet sich mit dem Schuh in der Hand von der Patientin ab, um einer anderen, verwirten Patientin hinterherzulaufen.)

S: Frau Maier, so warten Sie doch!

P: (hilflos rufend) „Schwester!“ (leiser) „Ich weiß wirklich gar nicht ...“ (Pause)

(S kommt zurück)

S: „So, Frau Mangold. Da wären wir wieder.“ (zieht ihr den Schuh an) „Ja, Sie werden jetzt gleich vorbereitet mit der Braunüle, weil Sie eine Röntgen-Darmuntersuchung bekommen.“

P: „Wie bitte?“

S: „Das hat Ihnen der Doktor doch sicher gesagt, oder?“

P: „Nein! Nicht richtig jedenfalls. Ich will das gar nicht. Sehen Sie“ (sie hält den verbundenen Arm hoch) „bei der letzten Untersuchung habe ich eine Entzündung bekommen. - Warum denn auch? - Mir hat keiner was gesagt! “

S: „Jetzt waschen wir uns erst einmal richtig." (S hilft P aufzustehen, um zum Waschbecken zu gehen $)^{5}$

\section{Das Bedrohliche im Antlitz der De-} menz

Insbesondere auf chirurgischen Stationen, in denen die prä- und postoperative Pflege im Vordergrund steht, wird das klinische Fachpersonal im Umgang mit Patienten mit Demenz nicht selten vor die zusätzliche Herausforderung gestellt, sich im pflegerischen Umgang mit den eigenen, meist unbewussten Ängsten konfrontiert zu sehen - und vor diesem Anblick zurückzuweichen.

Hier ist vor allem die Angst zu nennen, selbst gebrechlich und in hohem Maß von anderen abhängig zu werden, die Angst vor einem sich über lange Zeit hinziehenden Sterbeprozess, die Angst vor dem Tod an sich, und vielleicht auch die Angst, das eigene Leben nicht bewusst genug gelebt zu haben.

\section{Was soll ich tun?}

Ob wir einen menschenwürdigen Umgang insbesondere mit Patienten mit Demenz pflegen, hängt für den Sozialpsychologen und Psychogerontologen Tom Kitwood (1937-1998) ${ }^{6}$ wesentlich davon ab, ob es uns gelingt, „uns über unsere eigenen Ängste und Formen der Abwehr hinauszubewegen, so dass wahre Begegnung eintreten und lebensspendende Beziehungen wachsen können“, oder ob wir diese Menschen aus dem „Club der Personen“ ausschließen. Worauf Kitwood hinaus will, ist, dass es nicht nur im Hinblick auf die Bewältigung unserer Ängste, sondern auch hinsichtlich unseres Umgangs mit Patienten mit Demenz klüger wäre, wenn wir unseren Begriff der „Person“ im Sinne der Aufklärung neu überdenken würden.

\section{Die Fallgeschichte: 1. Szene (2. Teil) \\ - Schwester Maria}

(Dr. Wilken, Arzt (A), kommt plötzlich eilig ins Zimmer, wirkt gehetzt.)

A: „Schwester äh ...“(ihm fällt der Name nicht ein)

S: (lässt P auf das Bett zurückfallen, geht auf den Arzt zu, dem sie etwas sagen will, was P nicht hören soll)

S: „Dr. Wilken, ich glaube die Patientin..."

A: (fällt ihr ins Wort) „Was wollen Sie denn? Ich muss gleich zur Besprechung.“

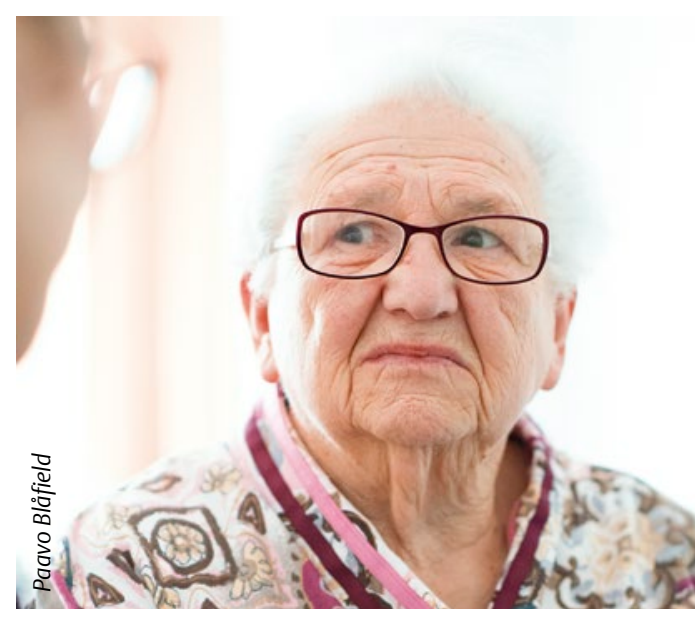

Menschen mit Demenz überfordern mit ihrem speziellen Pflegebedarf mitunter die Mitarbeiter in einem Akutkrankenhaus (Symbolbild).

S: „Ich denke, Frau Mangold ist nicht richtig aufgeklärt worden. Sie weiß gar nicht ..."

A: (unterbricht) „Selbstverständlich ist die Patientin aufgeklärt worden. - Was machen denn die Elektrolyte von der Frau Müller? Gibt es da schon Ergebnisse?“ (S zuckt mit den Schultern) „Hören Sie, ich brauche die Befunde spätestens in einer Stunde. Sorgen Sie dafür!" (A eilt aus dem Zimmer)

(S führt P hinter einen Paravent zum Waschen. Während des Waschens tönt es über einen Lautsprecher:) „Frau Mangold, bitte in die Röntgenabteilung, Frau Mangold, bitte in die Röntgenabteilung! “ ( $A$ stürzt ins Zimmer, sucht nach irgendetwas. Er verlässt den Raum und kommt erneut zurück, mit einem Set zum Anlegen der Braunüle. Er geht auf $P z u$. S tritt empört dazwischen.)

S: „Moment! Die Patientin ist noch dabei, sich zu waschen.“

A: „Haben Sie nicht gehört? Sie muss ins Röntgen!“ (A setzt sich vor P und greift zu ihrem Arm.)

A: „Ich brauch mal Ihren Arm.“ (Er legt den Venenzugang und steht danach wieder auf.) „Das wäre geschafft. Alles Weitere erledigt die Röntgenabteilung.“

P: „Aber warum? Ich will doch ...“

A: (unterbricht sie) „Wollen? - Gute Frau, was wollen Sie denn? - Sehen Sie, Sie haben ein Kolon-Karzinom. Wir müssen Sie so behandeln! Oder wollen Sie lieber sterben?" (Wartet keine Antwort ab und wendet sich zum Gehen.) 


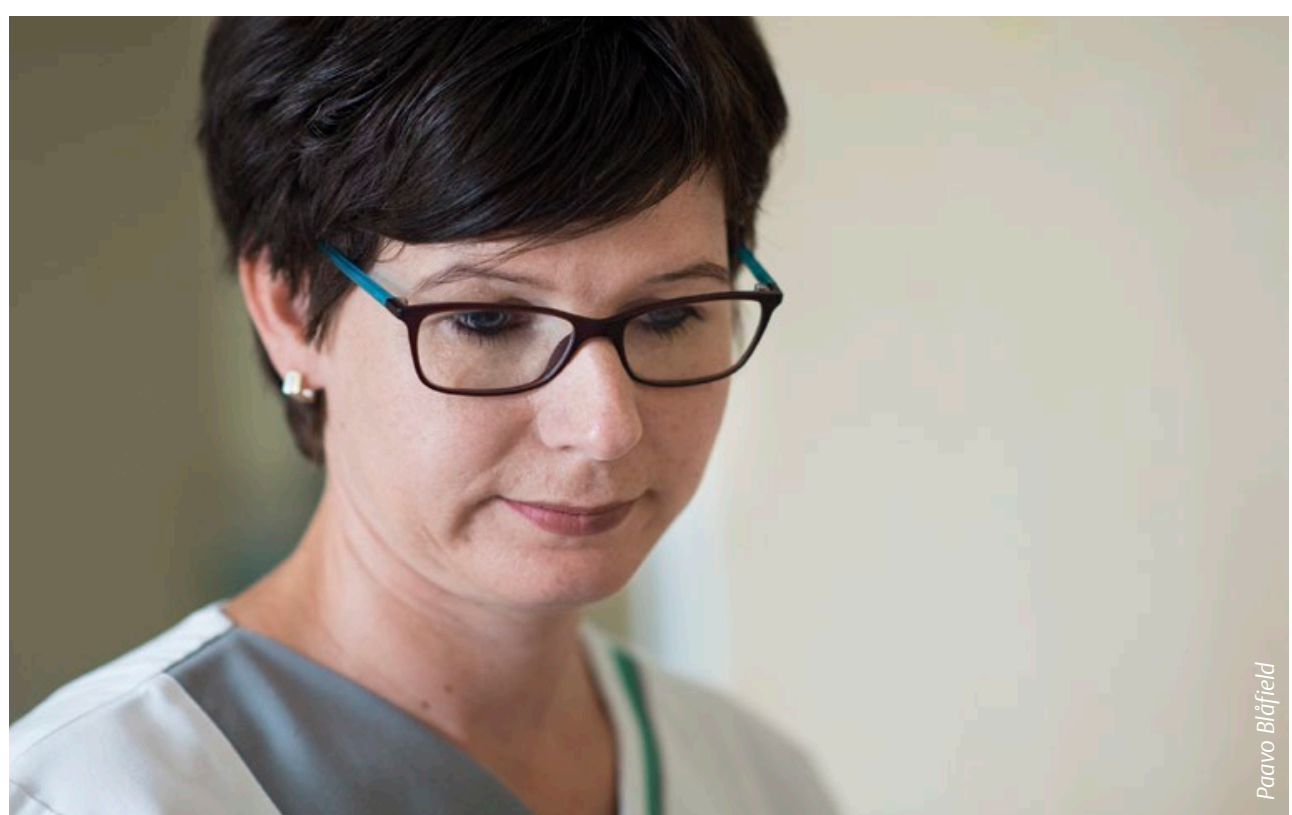

Schwester Maria spürt, dass etwas im Umgang mit Frau Mangold falsch läuft (Symbolbild).

P: (hilflos) „Nein, nein, ich weiß nicht ... Ich weiß doch gar nichts.“

(S geht an P vorbei, um einen Morgenmantel zu holen. P fasst nach dem Arm von $S$, die aber achtlos weitergeht. S hängt $P$ den Morgenmantel um und hilft ihr beim Aufstehen. Sie gehen zur Tür.)

S: „Sehen Sie. So ist es doch auch besser.“5

\section{„Die Person“ mit Demenz}

Für Kant ist der Mensch einzigartige „Person“, weil er keine austauschbare Sache ist, die diesem oder jenem Zweck als Mittel dient. Diese Würde, mehr zu sein, als ein bloßes Ding, gilt es im menschlichen Umgang miteinander zu pflegen. Doch der rationale Begriff der „Person“, mit dessen Hilfe die ausgehende Aufklärung Ende des 18. Jahrhunderts die Würde aller Menschen garantieren wollte und Worte wie Autonomie, Freiheit und Vernunft sind gegenwärtig nur noch Schatten im Hintergrund der modernen „Business-Kultur“, der es darum geht, Menschen mit ernsten Behinderungen aus dem „Club der Personen“ auszuschließen, indem alles dem Ideal des vernünftig denkenden und rational entscheidenden Menschen unterworfen wird.

Mit Kitwood können wir gegen diesen vereinseitigten Personen-Begriff seinen sozialpsychologischen Begriff von Personsein setzten: Person als ein Stand oder Status, der dem einzelnen Menschen im Kon- hung aus, die offen ist für die Begegnung mit dem anderen. Trete ich also an den Patienten mit Demenz mit einer Haltung heran, die den anderen zum „Dementen“ und damit zum bloßen Ding macht? Oder begegne ich ihm mit der Offenheit, in der er mir in seiner individuellen Einzigartigkeit als Person erscheinen kann?

Hier liegt eine enorme Verantwortung im pflegerischen Umgang mit Patienten mit Demenz: Da die Fähigkeit dieser Patienten zu öffentlichem Auftreten zunehmend nachlässt - und damit die Möglichkeit, mit entsprechend vielen anderen Personen Kontakte zu knüpfen, mit ihnen zu kommunizieren und zu interagieren -, ist ein Erspüren ihres Selbst für diese Personen meist nur noch mithilfe anderer Menschen möglich, die sich auf den Betroffenen wertschätzend und einfühlend einlassen können. ${ }^{9}$ Diese Verantwortung wird im Stationsalltag allerdings erst dann als praktische Fürsorge wirksam, wenn wir ein Gespür dafür entwickeln, was die wichtigsten Bedürfnisse dieser Menschen sein könnten. kein starrer Begriff mehr, der die rationale, vernünftige Seite des Menschen ins Zentrum setzt, sondern ein Netzwerk von Beziehungen zwischenmenschlichen Dialogs und gefühlsbetonten Miteinanderseins. Im Hintergrund dieses Begriffs steht nicht zuletzt der jüdische Religionsphilosoph Martin Buber (1878-1965), der in seinem 1932 erschienenen Hauptwerk „Ich und Du“8 von zwei Beziehungsarten im Rahmen menschlicher Interaktion ausgeht.

\section{Die zwei Arten des}

In-Beziehung-Tretens

Zum einen geht Buber von einer Ich-Es-Beziehung aus, die instrumentalisierend alles ihren Zwecken unterordnet und damit zum bloßen Objekt, zum Ding macht. Zum anderen geht er von einer Ich-Du-Bezie-

\section{Die Fallgeschichte: 2. Szene}

(S sitzt auf dem Stuhl und starrt ins Leere.)

S: (nachdenklich) „Also, das mit der Mangold. - So ganz in Ordnung war das ja wohl nicht. Die wollte schließlich nur wissen, was los ist mit ihr. - Wie sie meine Hand genommen hat. - Ich hätte sie besser informieren sollen!“(S geht.)

(A steht vor einem Kleiderschrank und zieht sich um.)

A: (zieht den Arztkittel aus und ein Jackett an.) „Na ja, hektisch war's ja schon heute. - Und diese Frau Mangold. - Eigentlich wollte ich den Job ja mal machen, um Menschen zu helfen. - Nur ..." (er sieht auf seine Armbanduhr) „pack ich das nicht, oder woran liegt das?“ (Er zögert kurz und verlässt dann rasch den Raum. $)^{5}$ 


\section{Identität und Einbeziehung}

Was unsere anfangs erwähnten Ängste um das eigene Selbst angeht, so empfiehlt uns der US-amerikanische Philosoph Richard Rorty (1931-2007), nicht länger von einem „Wesen“ des Selbst, einem "Ich“ oder einer "Seele“ zu sprechen, sondern - in ganz ähnlicher Weise wie Tom Kitwood - von einem „Beziehungsnetzwerk, das neu gewoben werden muss, ein Netzwerk, das die Zeit jeden Tag vergrößert". ${ }^{10}$ Da wir dem anderen allerdings die für dieses Netzwerk notwendigen „lebensspendenden Beziehungen“ und damit sein Selbst auch verweigern können, zeigt sich noch einmal sehr genau, mit welcher Haltung wir Patienten mit Demenz anhaltendenden Schaden zufügen können: indem wir ihnen zwei für ihr Personsein grundlegende Bedürfnisse verweigern: „Identität“ und „Einbeziehung“.

Unter „Identität“ können wir im Anschluss an Kitwood das Erkennen und Fühlen einer Kontinuität mit der Vergangenheit verstehen, die Möglichkeit, eine Geschichte von sich erzählen zu können und damit ein Selbst zu sein. Hier gründet nicht zuletzt die wichtige Bedeutung der gemeinsamen Biographiearbeit.

„Einbeziehung“ meint das - besonders bei Patienten mit Demenz ausgeprägte Bedürfnis, Teil einer Gruppe, einer Familie - bildlich gesprochen: Mitglied im „Club der Personen“ - zu sein, der einem Schutz und Sicherheit gewährt. Herausforderndes Verhalten, Anklammern und Umhergehen oder auch verschiedene Formen des Protests und der Zerrissenheit können möglicherweise Ausdruck eines solchen unbefriedigten Bedürfnisses sein.

\section{Fazit: Demenz ist nicht nur eine Krankheit}

Mit Kitwood können wir gegen die einseitige Betrachtung von Demenz als einem bloß neuropathologischen Geschehen ein Verständnis von Demenz setzen, das auch sozialpsychologische Faktoren und das gefühlsbetonte Erleben einer Person in sich vereinen kann.

Selbstverständlich bleiben medizinische Einsichten und die entsprechenden diagnostischen Rückschlüsse wichtig, nicht nur im Hinblick auf die Differenzialdiagnostik, sondern auch, da ganz spezifi-

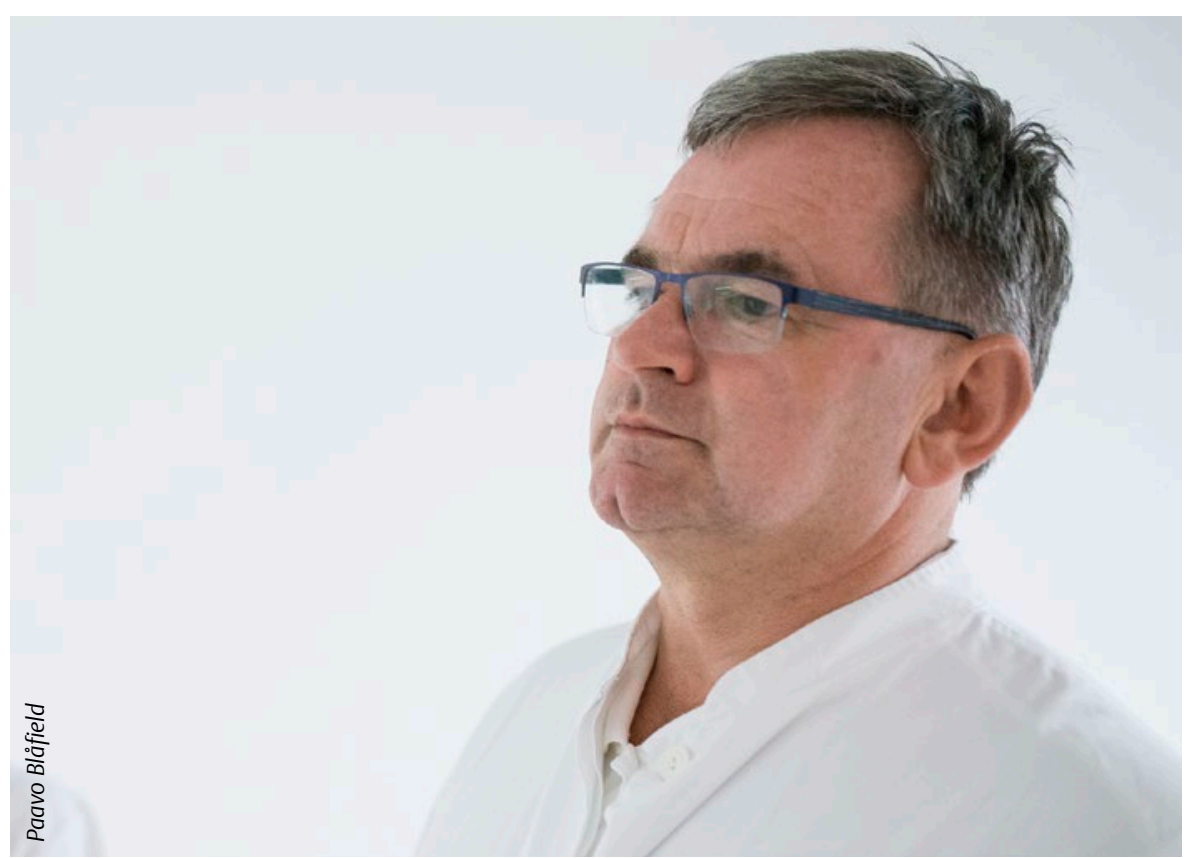

Auch Dr. Wilken hat seine Zweifel ... (Symbolbild).

sche Symptome (beispielsweise Vergesslichkeit und Sprachstörungen, motorische Störungen und schwere Verhaltensstörungen) ganz verschiedener Demenzformen die Pflegeplanung wesentlich beeinflussen und den Umgang mit Patienten mit Demenz bestimmen können. Den Fokus nur auf das neuropathologische Geschehen bzw. nur auf die „Krankheit“ zu zentrieren, erscheint dabei aus der Sicht einer person-zentrierten Pflege allerdings als Flucht vor der eigentlichen Fürsorge für das Personsein dieser Patienten.

\section{Literatur}

1 General Hospital Study - GHoSt. Zusammenfassung einer repräsentativen Studie zu kognitiven Störungen und Demenz in den Allgemeinkrankenhäusern von Baden-Württemberg und Bayern (2016). Robert Bosch Stiftung GmbH, Stuttgart. Im Internet: http://www.bosch-stiftung.de/content/language1/downloads/Studie_Demenz_im_ Akutkrankenhaus.pdf. Stand: 04.04.2016

2 Kleina T, Wingenfeld K. Die Versorgung demenzkranker älterer Menschen im Krankenhaus, Veröffentlichungsreihe des Instituts für Pflegewissenschaft an der Universität Bielefeld (IPW), 2007. Im Internet: https://www.uni-bielefeld.de/gesundhw/ag6/downloads/ipw-135.pdf; Stand: 04.04.2016

3 Saravay SM, Lavin M. Psychiatric comorbidity and length of stay in the general hospital - A critical review of outcome studies. Psychosomatics 1994; 35, 3: 233-252.

4 Quack E. Menschen mit Demenz im Krankenhaus. Im Spannungsfeld zwischen Systemlogik und Lebenswelt. Internationale Zeitschrift für Philosophie und Psychosomatik 2015; 2: 1-12. Im In- ternet: http://www.izpp.de/fileadmin/user_upload/Ausgabe_2_2015/Quack_IZPP_2_2015.pdf; Stand: 04.04.2016

5 Arbeitsgruppe „Pflege und Ethik“ der Akademie für Ethik in der Medizin e. V. „Für alle Fälle ...“ Arbeit mit Fallgeschichten in der Pflegeethik. Hannover: Brigitte Kunz Verlag; 2005

6 Kitwood T. Demenz. Der person-zentrierte Ansatz im Umgang mit verwirrten Menschen. Bern: Verlag Hans Huber; 2013

7 Kant I. Die Metaphysik der Sitten (1797). Berlin: de Gruyter; 1968

8 Buber M. Ich und Du. Stuttgart: reclam; 2008

9 Müller-Hergl C. Demenz zwischen Angst und Wohlbefinden: positive Personenarbeit und das Verfahren des Dementia Care Mapping. Demenz und Pflege 2008; 248-262

10 Rorty R. Kontingenz, Ironie und Solidarität. Frankfurt a. M.: Suhrkamp; 1992

\section{Autor}

Dr. Joachim Heil M.A.

Krankenpfleger, Studium der Philosophie, Dr. phil., Dozententätigkeit an der Johannes-Gutenberg-Universität Mainz und den Horst-Schmidt-Kliniken Wiesbaden. Zurzeit Mitarbeiter der Servicestelle für Patienten mit kognitiven Einschränkungen oder Demenz der Universitätsmedizin Mainz

joachim.heil@unimedizin-mainz.de

\section{Bibliografie}

DOI 10.1055/s-0043-110575

GGP 2017; 1: 62-65

(c) Georg Thieme Verlag KG

Stuttgart · New York · ISSN 1439-2569 